\title{
Applying Convex Optimal Power Flow to Combined Economic and Emission Dispatch
}

\author{
Zhao Yuan, Mohammad Reza Hesamzadeh \\ Department of Electrical Power and Energy Systems, KTH Royal Institute of Technology, Stockholm, Sweden \\ Email: yuanzhao@kth.se
}

Received 24 May 2016; accepted 18 July 2016; published 21 July 2016

\begin{abstract}
This paper addresses the problem of reducing $\mathrm{CO}_{2}$ emissions by applying convex optimal power flow model to the combined economic and emission dispatch problem. The large amount of $\mathrm{CO}_{2}$ emissions in the power industry is a major source of global warming effect. An efficient and economic approach to reduce $\mathrm{CO}_{2}$ emissions is to formulate the emission reduction problem as emission dispatch problem and combined with power system economic dispatch (ED). Because the traditional optimal power flow (OPF) model used by the economic dispatch is nonlinear and nonconvex, current nonlinear solvers are not able to find the global optimal solutions. In this paper, we use the convex optimal power flow model to formulate the combined economic and emission dispatch problem. The advantage of using convex power flow model is that global optimal solutions can be obtained by using mature industrial strength nonlinear solvers such as MOSEK. Numerical results of various IEEE power network test cases confirm the feasibility and advantage of convex combined economic and emission dispatch (CCEED).
\end{abstract}

\section{Keywords}

$\mathrm{CO}_{2}$, Combined Econnomic and Emission Dispatch, Convex Optimal Power Flow

\section{Introduction}

Electricity generation from fossil fuel based power plants is one major source of greenhouse gas emissions, [1] [2]. According to reference [1], 37.5\% of total $\mathrm{CO}_{2}$ emissions are from the global electricity supply sector. The current approaches to mitigate $\mathrm{CO}_{2}$ emissions include more efficient fossil fuel conversion, switching to lowcarbon energy resources, decarbonisation of fuels and nuclear power, [1]. It is critical to analyze cost and performance of different $\mathrm{CO}_{2}$ reduction or capture approaches, [3]. The combined economic and emission dispatch (CEED) considered in this paper can be categorized to efficient management or market arrangement to reduce $\mathrm{CO}_{2}$ emissions. Reference [4] formulate the combined economic and emission dispatch (CEED) by converting the bi-objective optimization problem to weighted single objective optimization. The Gravitational Search Algorithm (GSA) is used to solve the nonconvex CEED model in [5]. Reference [6] proposes to apply nondominated sorting genetic algorithm-II to find pareto-optimal solutions of the formulated dynamic economic emission dispatch problem in one single run. Reference [7] deploys Particle Swarm Optimization (RPSO) to solve CEED in 
distribution system. The core mathematical model in the CEED is optimal power flow (OPF). OPF solves a system level objective constrained by power network physical and operational conditions, [7-12]. Current methods to solve optimal power flow problem include using DC OPF model and nonconvex AC OPF model. DC OPF assumes voltage magnitude of all buses or nodes in the networks are fixed to 1 per unit value and the voltage phase angle difference across each line is small enough so that $\sin \left(\theta_{l}\right) \approx \theta_{l}$, [7]. DC OPF neglect resistance parameter of power transmission lines. These assumptions are valid for most high voltage power transmission networks. But in low voltage power distribution networks, DC OPF is not valid, [8]. Even for high voltage power transmission networks, it is still possible that assumptions used in DC OPF fail to meet real world situations, [8-9]. For the nonconvex AC OPF, current nonlinear programming solvers are only able to find local optimal solutions, [10]. There is no guarantee to obtain global optimal by using nonconvex AC OPF. Though branch and bound is promising method to find global optimal solution of nonconvex AC OPF, the computation time of branch and bound method is too long to be implemented in practice especially for large scale networks, [11]. The advantage of convex methods in CCEED is that global optimal solutions can be guaranteed by mature nonlinear programming solvers such as MOSEK, [13].

\section{Convex Combined Economic and Emission Dispatch}

For ease of illustration, the nonconvex AC optimal power flow model used in the economic dispatch problem is reformulated here as (1)-(11). The formulations in (1)-(11) are based on transmission line sending end power injection variables. The advantage of including voltage phase angle explicitly in the formulation (1)-(11) is that we can obtain voltage phase angle solutions directly by solving this model.

$$
\begin{aligned}
& \text { Minimize } \quad f=f\left(p_{i}, q_{i}, p_{o l}, q_{o l}\right) \\
& \text { subject to } \quad p_{i}-D_{i}^{p}=\sum_{l}\left(\mathrm{~A}_{i l} \cdot p_{s l}\right)-\sum_{l}\left(B_{i l} \cdot p_{o l}\right) \\
& q_{i}-D_{i}^{q}=\sum_{l}\left(\mathrm{~A}_{i l} \cdot q_{s l}\right)-\sum_{l}\left(B_{i l} \cdot q_{o l}\right) \\
& V_{s l}-V_{r l}=2 R_{l} p_{s l}+2 X_{l} q_{s l}-R_{l} p_{o l}-X_{l} q_{o l} \\
& v_{s l} V_{r l} \sin \left(\theta_{l}\right)=X_{l} p_{s l}-R_{l} q_{s l} \\
& p_{o l}^{\max } \geq p_{o l}=\frac{p_{s l}^{2}+q_{s l}^{2}}{V_{s l}} R_{l} \\
& X_{l} p_{o l}=R_{l} q_{o l} \\
& p_{i}^{\min } \leq p_{i} \leq p_{i}^{\max } \\
& q_{i}^{\min } \leq q_{i} \leq q_{i}^{\max } \\
& V_{n}^{\min } \leq V_{n} \leq V_{n}^{\max } \\
& \theta_{l}^{\min } \leq \theta_{l} \leq \theta_{l}^{\max }
\end{aligned}
$$

where $i$ is the index of bus or node in the power network. $l$ is the index of transmission line or distribution line. $f$ in equation (1) is the objective function. Note that the variables in the objective function (1) are only for purpose of illustration. The objective function of OPF can be formulated in any way based on the operation targets. $\quad p_{i}$ is the active power injection at bus $i . D_{i}^{p}$ is the active power load at bus $i . D_{i}^{q}$ is the reactive power injection at bus $i . D_{i}^{p}$ and $D_{i}^{q}$ are power network operation parameters. $p_{s l}$ is the active power transmitted at the sending end of line $l . q_{s l}$ is the reactive power transmitted at the sending end of line $l$. $A_{i l}$ is the network topology matrix with $A_{i l}=1$ if node $i$ is at the sending end of line $l, A_{i l}=-1$ if node $i$ is at the receiving end of line $l$ and $A_{i l}=0$ if node $i$ is not connected with line $l \cdot B_{i l}$ is the network topology matrix with $B_{i l}=1$ if node $i$ is at the receiving end of line $l$ and $B_{i l}=0$ if node $i$ is at the re- 
ceiving end of line $l$ or not connected with line $l . A_{i l}$ and $B_{i l}$ are network topology parameters. $V_{s l}=v_{s l}^{2}$ is the voltage magnitude square at the sending end of line $l . V_{r l}=v_{r l}^{2}$ is the voltage magnitude square at the receiving end of line $l . v_{s l}$ is the voltage magnitude at the sending end of line $l . v_{r l}$ is the voltage magnitude at the receiving end of line $l . R_{l}$ is the resistance of line $l . X_{l}$ is the reactance of line $l . R_{l}$ and $X_{l}$ are network physical parameters. $p_{o l}$ is active power loss of line $l . q_{o l}$ is reactive power loss of line $l$. $\theta_{l}$ is voltage phase angle difference of line $l . p_{o l}^{\max }$ is the upper bound of active power loss. Equation (2) represents the active power balance for each bus. Equation (3) represents the reactive power balance for each bus. Constraint (6) is active power loss constraint. Constraints (8)-(11) are bounds for OPF variables. Equation (5) and (6) are nonconvex. We approximate equation (5) in the original AC optimal power flow model by equation (12).

$$
\theta_{l}=X_{l} p_{s l}-R_{l} q_{s l}
$$

Apparently, the underlined assumptions for Equation (12) to be valid are (a)-(b).

(a) $v_{s l} v_{r l} \approx 1$

(b) $\sin \left(\theta_{l}\right) \approx \theta_{l}$

Because the operation constraint for voltage magnitude (per unit value) is generally $0.9 \leq v_{n} \leq 1.1$ and the voltage phase angle difference is generally small along transmission lines, assumptions (a)-(b) are valid in most situations during power system real operations.

Equation (6) is convexified by rotated cone expressed in (13).

$$
p_{o l}^{\max } \geq p_{o l} \geq \frac{p_{s l}^{2}+q_{s l}^{2}}{V_{s l}} R_{l}
$$

The method used in (13) to make equation (6) convex is relaxation. In this case, we actually relax quadratic equality constraint to quadratic inequality constraint. The tightness of this relaxation can be guaranteed by implicitly include power loss component in the objective function. This has been proved by numerical results, [13]. Thus, if we use OPF model [(1)-(4), (7)-(13)] instead of [(1)-(11)], we can obtain a convex OPF model. This model can be used to formulate combined economic and emission dispatch problem. Generally the objective function $f$ in (1) is the cost of active power generation from all the generators formulated as quadratic function:

$$
f=\sum_{i}\left(c_{i, 2} p_{i}^{2}+c_{i, 1} p_{i}\right)
$$

where $c_{i, 2}$ and $c_{i, 1}$ are cost coefficients for active power generation. These parameters can be obtained directly from MATPOWER, [14].

To include the $\mathrm{CO}_{2}$ emission reduction target to the economic dispatch problem, we formulate the combined economic and emission dispatch by assigning weights to the cost and $\mathrm{CO}_{2}$ emission parts in the objective function as follows (15).

$$
f=w_{1}\left[\sum_{i}\left(c_{i, 2} p_{i}^{2}+c_{i, 1} p_{i}\right)\right]+w_{2}\left(\sum_{i} \alpha_{i} p_{i}\right)
$$

where $w_{1}, w_{2} \in[0,1]$ are weights for corresponding terms in the objective function satisfying $w_{1}+w_{2}=1$. These weights can be adjusted by the system operator depending on the importance of cost and $\mathrm{CO}_{2}$ emission. Obviously, when $w_{1}=1$ and $w_{2}=0$ the CCEED problem is reduced to power system economic dispatch. When $w_{1}=0$ and $w_{2}=1$ the CCEED problem is reduced to CO2 emission minimization without considering economic cost. $\alpha_{i}$ in Equation (15) is the $\mathrm{CO}_{2}$ emission coefficient for fossil fuel power plant. It is calculated by Equation (16). The results of Equation (16) are listed in Table 1. Emission factor and power plant efficiency data in Table 1 are from reference [15]. The power generation technology for steam coal, fuel oil and diesel oil is assumed to be simple cycle combustion turbine. For natural gas, the combustion turbine is assumed to be combined cycle based technology.

$$
\alpha_{i}=\frac{\text { Emission Factor }}{\text { Power Plant Efficiency }}
$$


Table 1. $\mathrm{CO}_{2}$ Emission Coefficients for Different Fuel Types.

\begin{tabular}{cccc}
\hline Fuel Type & Emission Factor [tC/MWh] & Power Plant Efficiency & Emission Coefficient $\alpha_{i}[\mathrm{tC} / \mathrm{MWh}]$ \\
\hline Steam Coal & 0.9288 & $37 \%$ (Simple Cycle) & 2.5103 \\
Fuel Oil & 0.7596 & $30 \%$ (Simple Cycle) & 2.532 \\
Diesel Oil & 0.7272 & $30 \%$ (Simple Cycle) & 2.424 \\
Natural Gas & 0.5508 & $50 \%$ (Combined Cycle) & 1.1016 \\
\hline
\end{tabular}

Table 2. IEEE14 Test Case CCEED Results.

\begin{tabular}{cccc}
\hline$w_{1}$ & $w_{2}$ & cost $[\$]$ & $\mathrm{CO}_{2}$ Emission $\left[\mathrm{tCO}_{2}\right]$ \\
\hline 1 & 0 & 8078.84 & 2422.63 \\
0.8 & 0.2 & 8095.65 & 2295.04 \\
0.6 & 0.4 & 8171.71 & 2117.05 \\
0.5 & 0.5 & 8253.02 & 2019.42 \\
0.4 & 0.6 & 8431.85 & 1876.23 \\
0.2 & 0.8 & 9607.23 & 1390.22 \\
0 & 1 & 465485.22 & 1346.87 \\
\hline
\end{tabular}

Table 3. IEEE57 Test Case CCEED Results.

\begin{tabular}{cccc}
\hline$w_{1}$ & $w_{2}$ & cost $[\$]$ & $\mathrm{CO}_{2}$ Emission $\left[\mathrm{tCO}_{2}\right]$ \\
\hline 1 & 0 & 41696.94 & 9139.32 \\
0.8 & 0.2 & 41707.77 & 9040.54 \\
0.6 & 0.4 & 41771.31 & 8901.59 \\
0.5 & 0.5 & 41801.98 & 8859.66 \\
0.4 & 0.6 & 41802.98 & 8858.86 \\
0.2 & 0.8 & 41811.24 & 8855.75 \\
0 & 1 & 460648.82 & 8845.95 \\
\hline
\end{tabular}

\section{Test Results}

The CCEED model is coded in the General Algebraic Modeling System (GAMS). MOSEK solver in GAMS is used to solve the CCEED model. Generator and IEEE test network parameters in MATPOWER [14] are directly used. To differentiate the $\mathrm{CO} 2$ emission parameters for different generators, we assign the four different fuel types evenly to different generators. A laptop running on windows 7 64-bit operating system with Intel i7-2760QM 2.4 GHz CPU and 8G RAM is deployed. The CCEED results for IEEE14, IEEE57 and IEEE118 [16] test cases are listed in Tables 2-4. The performance of CCEED is demonstrated by varying weights parameters $\left(w_{1}, w_{2}\right)$ in the objective function (15). The CPU computation time of all test cases in GAMS is less than 0.1 second.

It can be observed from Tables 2-4 that with the increase of weights $w_{2}$ on the $\mathrm{CO}_{2}$ emission term of the objective function in CEEED, the $\mathrm{CO}_{2}$ emission is decreasing. The cost of power production increase sharply when $w_{1}=0$ and $w_{2}=1$. This means to minimize $\mathrm{CO}_{2}$ emissions without considering economic cost is not feasible in reality. As a good compromise, $w_{1}=0.5$ and $w_{2}=0.5$ can be regarded one cost effective arrangement to reduce $\mathrm{CO}_{2}$ emissions. 
Table 4. IEEE118 Test Case CCEED Results.

\begin{tabular}{cccc}
\hline$w_{1}$ & $w_{2}$ & cost $[\$]$ & $\mathrm{CO}_{2}$ Emission $\left[\mathrm{tCO}_{2}\right]$ \\
\hline 1 & 0 & 129619.53 & 37189.08 \\
0.8 & 0.2 & 129672.93 & 36793.08 \\
0.6 & 0.4 & 130094.74 & 35917.63 \\
0.5 & 0.5 & 130630.21 & 35274.08 \\
0.4 & 0.6 & 131444.34 & 34613.92 \\
0.2 & 0.8 & 136241.22 & 32689.10 \\
0 & 1 & 879402.23 & 31039.88 \\
\hline
\end{tabular}

\section{Conclusion}

In this paper, we prove the feasibility of using convex optimal power flow model to solve the combined economic and emission dispatch problem. The original nonconvex optimal power flow model is approximated and relaxed by mathematical techniques. The underlined assumptions of these approximations are explained in detail. The CCEED problem is then formulated by assigning weights to the power generation cost minimization objective and $\mathrm{CO}_{2}$ emission minimization objective. The CCEED model is built in GAMS platform and solved by MOSEK. Numerical results from IEEE14, IEEE57 and IEEE118 test cases show that CCEED can be solved efficiently. By adjusting the weights for cost and $\mathrm{CO}_{2}$ emissions in the objective of CCEED, a compromise between power generation cost and $\mathrm{CO}_{2}$ reduction can be achieved. Instead of nonconvex model, our convex model can guarantee global optimal solutions. Though we demonstrate the usefulness of CCEED by reducing $\mathrm{CO}_{2}$ emissions here, the potential applications of CCEED can be extended to reducing other green-house gas emissions or air pollutants.

\section{References}

[1] Sims, R.E.H., Rogner, H.-H. and Gregory, K. (2003) Carbon Emission and Mitigation Cost Comparisons between Fossil Fuel, Nuclear and Renewable Energy Resources for Electricity Generation. Energy Policy, 31, 1315-1326.

[2] IPCC, Climate Change 2014 Mitigation of Climate Change Working Group III Contribution to the Fifth Assessment Report of the Intergovernmental Panel on Climate Change, Cambridge University Press, England.

[3] Rubin, E.S., Chen, C. and Rao, A.B. (2007) Cost and Performance of Fossil Fuel Power Plants with $\mathrm{CO}_{2}$ Capture and Storage. Energy Policy, 35, 4444-4454.

[4] Basu, M. (2008) Dynamic Economic Emission Dispatch Using Nondominated Sorting Genetic Algorithm-II. International Journal of Electrical Power \& Energy Systems, 30, 140-149. http://dx.doi.org/10.1016/j.ijepes.2007.06.009

[5] Güvenç, U., Sönmez, Y., Duman, S. and Yörükeren, N. (2012) Combined Economic and Emission Dispatch Solution Using Gravitational Search Algorithm. Scientia Iranica, 19, 1754-1762. http://dx.doi.org/10.1016/j.scient.2012.02.030

[6] Swarnkar, K.K., Wadhwani, S. and Wadhwani, A.K. (2009) Optimal Power Flow of Large Distribution System Solution for Combined Economic Emission Dispatch Problem using Partical Swarm Optimization. International Conference on Power Systems, 2009. ICPS '09, Kharagpur, 1-5. http://dx.doi.org/10.1109/icpws.2009.5442713

[7] Shoults, R.R. and Sun, D.T. (1982) Optimal Power Flow Based Upon P-Q Decomposition. IEEE Transactions on Power Apparatus and Systems, PAS-101, 397-405. http://dx.doi.org/10.1109/TPAS.1982.317120

[8] Purchala, K., Meeus, L., Van Dommelen, D. and Belmans, R. (2005) Usefulness of DC Power Flow for Active Power Flow Analysis. IEEE Power Engineering Society General Meeting, 1, 454-459. http://dx.doi.org/10.1109/pes.2005.1489581

[9] Capitanescu, F. (2016) Critical Review of Recent Advances and Further Developments Needed in AC Optimal Power Flow. Electric Power Systems Research, 136, 57-68. http://dx.doi.org/10.1016/j.epsr.2016.02.008

[10] Paper, A.S., Castillo, A., O’neill, R.P., Castillo, A. and O’neill, R.P. (2013) Survey of Approaches to Solving the ACOPF Optimal Power Flow Paper 4, US Federal Energy Regulatory Commission, Tech. Rep,

[11] Gopalakrishnan, A., Raghunathan, A.U., Nikovski, D. and Biegler, L.T. (2012) Global Optimization of Optimal Power Flow Using a Branch \& Bound Algorithm. 2012 50th Annual Allerton Conference on Communication, Control, and 
Computing (Allerton), Monticello, 609-616. http://dx.doi.org/10.1109/allerton.2012.6483274

[12] Baradar, M. and Hesamzadeh, M.R. (2015) AC Power Flow Representation in Conic Format. IEEE Transactions on Power Systems, 30, 546-547. http://dx.doi.org/10.1109/TPWRS.2014.2326980

[13] MOSEK Manual, MOSEK ApS, C/O Symbion Science Park, Fruebjergvej 3, Box 16, 2100 Copenhagen, Denmark.

[14] Zimmerman, R.D., Murillo-Sanchez, C.E. and Thomas, R.J. (2011) MATPOWER: Steady-State Operations, Planning, and Analysis Tools for Power Systems Research and Education. IEEE Transactions on Power Systems, 26, 12-19. http://dx.doi.org/10.1109/TPWRS.2010.2051168

[15] dos Santos, M.A., Rosa, L.P., Sikar, B., Sikar, E. and dos Santos, E.O. (2006) Gross Greenhouse Gas Fluxes from Hydro-Power Reservoir Compared to Thermo-Power Plants. Energy Policy, 34, 481-488. http://dx.doi.org/10.1016/j.enpol.2004.06.015

[16] The University of Washington Power Systems Test Case Archive. http://www.ee.washington.edu/research/pstca/

\section{Submit or recommend next manuscript to SCIRP and we will provide best service for you:}

Accepting pre-submission inquiries through Email, Facebook, LinkedIn, Twitter, etc.

A wide selection of journals (inclusive of 9 subjects, more than 200 journals)

Providing 24-hour high-quality service

User-friendly online submission system

Fair and swift peer-review system

Efficient typesetting and proofreading procedure

Display of the result of downloads and visits, as well as the number of cited articles

Maximum dissemination of your research work

Submit your manuscript at: http://papersubmission.scirp.org/ 